\title{
Review: evidence does not support an association between allergen levels and risk for asthma
}

\author{
Pearce N, Douwes J, Beasley R. Is allergen exposure the major primary cause of asthma? Thorax 2000 May;55:424-31.
}

QUESTION: Is allergen exposure the major primary cause of asthma?

\section{Data source}

Medline search (from 1980).

\section{Study selection}

English language studies were selected if they were longitudinal or cross sectional studies of the relation between allergens and asthma. The review focused on primary causation studies that reported the amount of allergen exposure. Studies that used bronchial hyperresponsiveness in the definition of asthma were excluded. Studies of selected populations were used when no information from population based studies was available.

\section{Data extraction}

Data were extracted on age, allergen levels, asthma prevalence, definition of asthma, and population attributable risk.

\section{Main results}

Results are reported for longitudinal studies only. No population based studies were found on the relation between allergen exposure during infancy and asthma risk after the age of 6 years. In 1 randomised controlled trial, 160 infants with a family history of atopy were allocated to prophylaxis (avoidance of house dust mite allergen and allergenic food) or no prophylaxis. At 12 months, asthma ( $\geqslant 3$ separate episodes of cough and wheezing) was more common in the control group than in the prophylaxis group (odds ratio [OR] 4.1, 95\% CI 1.1 to 15.5), but the difference was not statistically significant at 2 or 4 years. 2 cohort studies did not show statistically significant associations between allergen exposure and asthma risk in children who had a family history of allergy and who were followed beyond the age of 6 years. In 2 population based cohort studies that followed infants for $2 \mu \mathrm{g} / \mathrm{g}$ of $\operatorname{Der} p 1$ and wheeze and prolonged cough (adjusted OR 4.8, CI 1.1 to 21.1); the association was not statistically significant for exposure to $>5 \mu \mathrm{g} / \mathrm{g}$ of $\operatorname{Der} p 1$. In 1 study of 499 children whose parents had asthma, allergies, or both, exposure to cockroach allergen levels $>0.05 \mathrm{U} / \mathrm{g}$ in the first 3 months of life was associated with repeated wheeze in the first year of life (relative risk 1.63, CI 1.05 to 2.55); no association existed between repeated wheeze and dog and cat allergen levels.

\section{Conclusions}

No long term, population based, cohort studies have examined the relation between allergen levels and risk for asthma. An association between allergen levels and asthma has not been shown in short term, population based cohort studies or long term cohort studies in selected populations.

\section{COMMENTARY}

Genetic factors and outdoor pollution are unlikely to explain the differences in the prevalence of childhood asthma over time and among countries or the development of asthma and wheezing with migration, such as from China to Australia. Diet and allergens are potential explanatory factors. While evidence for a role of diet gathers strength, ${ }^{1}$ it is challenging to reconcile the differing findings of recent reviews of allergen exposure in primary asthma causation.

Peat and $\mathrm{Li}$ recently concluded that the available evidence for a role of house dust mite allergens in asthma causation met all the necessary Bradford Hill causation criteria. ${ }^{2}$ Pearce et al, however, conclude that such evidence is lacking, having focused mainly on longitudinal studies that evaluated primary asthma causation in infants with quantitative allergen exposure estimates and excluded studies that evaluated bronchial hyperreactivity, migratory studies, and secondary causation studies. Few studies met these criteria, and they had a limited range of allergen measure and categorical exposure scoring methods. The exclusion of secondary causation studies results in a focused review, although an "increase" in asthma may result partly from either increased wheezing in allergic people or an overall increase in allergy from immune system changes that might be related to diet, broad spectrum antibiotics, early childhood vaccination, or reduced infections. ${ }^{3}$ Reduced bronchial hyperreactivity with prolonged allergen avoidance suggests that allergen avoidance should be considered, ${ }^{4}$ although this is a secondary rather than primary causation issue.

What should a clinician recommend? Studies that meet the Bradford Hill criteria provide varying levels of evidence for some simple measures. Apart from reduction in bedroom carpets, other measures include avoiding passive smoking and unflued gas appliances, ${ }^{25}$ increasing fish and vegetable intake, and breast feeding for $\geqslant 3$ months. ${ }^{12}$ Asthma is a complex multifactorial disease, and the effectiveness of such interventions varies. Longitudinal studies that include evaluation of diet, allergies, indoor environment, potential confounders, and interactions are needed. Brian J Smith, MBBS North West Adelaide Health Service Richard Ruffin, MD University of Adelaide Woodville, South Australia, Australia

1 Hijazi N, Abalkhail B, Seaton A. Diet and childhood asthma in a society in transition: a study in urban and rural Saudi Arabia. Thorax 2000;55:775-9.

2 Peat JK, Li J. Reversing the trend: reducing the prevalence of asthma. JAllergy Clin Immunol 1999;103:1-10.

3 Platts-Mills TA, Blumenthal K, Perzanowski M, et al. Determinants of clinical allergic disease. The relevance of indoor allergens to the increase in asthma. Am J Respir Crit Care Med 2000;162:S128-33.

4 Platts-Mills TA, Tovey ER, Mitchell EB, et al. Reduction of bronchial hyperreactivity during prolonged allergen avoidance. Lancet 1982;2:675-8.

5 Smith BJ, Nitschke M, Pilottoe LS, et al. Health effects of daily indoor nitrogen dioxide in people with asthma. Eur Resp J 2000;16:879-85. 